\title{
Effects of inappropriate empirical antibiotic therapy on mortality in patients with healthcare-associated methicillin-resistant Staphylococcus aureus bacteremia: a propensity-matched analysis
}

Young Kyung Yoon ${ }^{1}$, Dae Won Park', Jang Wook Sohn', Hyo Youl Kim², Yeon-Sook Kim³ , Chang-Seop Lee, Mi Suk Lee ${ }^{5}$, Seong-Yeol Ryu ${ }^{6}$, Hee-Chang Jang ${ }^{7}$, Young Ju Choi ${ }^{8}$, Cheol-In Kang ${ }^{9}$, Hee Jung Choi ${ }^{10}$, Seung Soon Lee ${ }^{11}$, Shin Woo Kim² ${ }^{12}$, Sang $\|$ Kim$^{13}$, Eu Suk Kim ${ }^{14}$, Jeong Yeon Kim ${ }^{15}$, Kyung Sook Yang ${ }^{16}$, Kyong Ran Peck ${ }^{9}$ and Min Ja Kim ${ }^{1 *}$ (D)

\begin{abstract}
Background: The purported value of empirical therapy to cover methicillin-resistant Staphylococcus aureus (MRSA) has been debated for decades. The purpose of this study was to evaluate the effects of inappropriate empirical antibiotic therapy on clinical outcomes in patients with healthcare-associated MRSA bacteremia (HA-MRSAB).

Methods: A prospective, multicenter, observational study was conducted in 15 teaching hospitals in the Republic of Korea from February 2010 to July 2011. The study subjects included adult patients with HA-MRSAB. Covariate adjustment using the propensity score was performed to control for bias in treatment assignment. The predictors of in-hospital mortality were determined by multivariate logistic regression analyses.

Results: In total, 345 patients with HA-MRSAB were analyzed. The overall in-hospital mortality rate was $33.0 \%$. Appropriate empirical antibiotic therapy was given to 154 (44.6\%) patients. The vancomycin minimum inhibitory concentrations of the MRSA isolates ranged from 0.5 to $2 \mathrm{mg} / \mathrm{L}$ by E-test. There was no significant difference in mortality between propensity-matched patient pairs receiving inappropriate or appropriate empirical antibiotics (odds ratio $[\mathrm{OR}]=1.20 ; 95 \%$ confidence interval $[\mathrm{Cl}]=0.71-2.03$ ). Among patients with severe sepsis or septic shock, there was no significant difference in mortality between the treatment groups. In multivariate analyses, severe sepsis or septic shock (OR $=5.45 ; 95 \% \mathrm{Cl}=2.14-13.87)$, Charlson's comorbidity index (per 1-point increment; $\mathrm{OR}=1$. $52 ; 95 \% \mathrm{Cl}=1.27-1.83)$, and prior receipt of glycopeptides $(\mathrm{OR}=3.24 ; 95 \% \mathrm{Cl}=1.08-9.67)$ were independent risk factors for mortality.
\end{abstract}

Conclusion: Inappropriate empirical antibiotic therapy was not associated with clinical outcome in patients with HA-MRSAB. Prudent use of empirical glycopeptide therapy should be justified even in hospitals with high MRSA prevalence.

Keywords: Methicillin-resistant Staphylococcus aureus, Bacteremia, Risk factors, Treatment outcome, Anti-bacterial agents

\footnotetext{
* Correspondence: macropha@korea.ac.kr

'Department of Internal Medicine, Korea University College of Medicine,

Seoul, Republic of Korea

Full list of author information is available at the end of the article
} 


\section{Background}

Methicillin-resistant Staphylococcus aureus (MRSA) has been a major cause of healthcare-associated bacteremia $[1,2]$. MRSA bacteremia (MRSAB), with a mortality rate as high as $40 \%$, is a grave concern because a clinical cure may not be achieved using standard therapy in some cases [3-6]. Evidence-based therapy and the identification of mortality-related risk factors for MRSAB continue to represent significant clinical challenges for clinicians.

Previous studies determined the independent predictors of mortality among patients with MRSAB, including old age ( $\geq 60$ years), underlying cardiac diseases, a higher Charlson's comorbidity index, pneumonia, septic shock, metastatic infection, non-eradicable foci, and higher vancomycin minimum inhibitory concentrations (MICs) of the MRSA isolates [7-9]. However, studies evaluating the presence of relationships between clinical outcome and inappropriate empirical antibiotic therapies in patients with MRSAB have yielded conflicting results [10-16]. The conflicting results on the benefits of early empirical antibiotic therapy are probably due to differing definitions of "inappropriate" therapy on the basis of in vitro susceptibility data, impact of potentially confounding variables, and selection or information biases such as the baseline severity of illness $[17,18]$. The definition and criteria items used to denote the appropriate antibiotic therapy should include the criterion matching the in vitro susceptibility and the timing of the administration of the antibiotics as well as their optimal dose and usage $[19,20]$. A recent meta-analysis involving 510 MRSAB episodes demonstrated an overall 2 -fold increased survival benefit with the administration of appropriate empirical therapy for MRSAB episodes, but there are some problems with heterogeneity across the studies [21]. In the meanwhile, the prudent use of glycopeptides has been an important component of hospital antimicrobial stewardship programs to contain the emergence of glycopeptide resistance, especially in high-prescribing countries with a high prevalence of MRSA. Thus, empirical glycopeptides use for MRSAB should be guided on the basis of scientific data.

The purpose of this study was to assess the influence of inappropriate empirical antimicrobial therapy on mortality in patients with healthcare associated-MRSAB (HA-MRSAB) in a hospital setting in which the prevalence of MRSA was high.

\section{Methods}

\section{Study design and setting}

A prospective, multi-center, observational study was conducted to compare clinical outcomes between patients receiving inappropriate or appropriate antibiotics for the treatment of HA-MRSAB in 15 teaching hospitals in the
Republic of Korea from February 2010 to July 2011. This cross-sectional study was performed in accordance with the Strengthening the Reporting of Observational Studies in Epidemiology (STROBE) guidelines. Two analytical strategies were used: (1) for the non-matched case-control study $(n=345)$, the clinical outcomes of 154 patients who received appropriate empirical antibiotic treatment was compared with those of 191 patients who received inappropriate treatment; and (2) for the propensity scorematched (1:1) case-control study, the outcome was compared between 127 pairs of patients who received inappropriate or appropriate empirical antibiotics. Cases were defined as the patients treated with inappropriate empirical antibiotics, and controls were defined as those with HA-MRSAB who were treated with appropriate empirical antibiotics. The primary endpoint was all-cause in-hospital mortality. The secondary endpoints were mortality attributable to MRSAB, persistent fever, and persistent MRSAB.

All 15 participating hospitals have long had a high prevalence of MRSA, which accounted for about approximately $70 \%$ of all $S$. aureus isolates. Clinical data were collected at each participating site via a standardized web-based case report form. A representative infectious disease specialist from each site prospectively reviewed and interpreted clinical data for the enrolled patients. A trough serum concentration of vancomycin was measured routinely in 13 of the 15 participating hospitals in this study.

\section{Study population}

Study subjects included hospitalized adult patients (aged $\geq 18$ years) with HA-MRSAB who had $\geq 1$ positive blood culture for MRSA. Our study excluded the subjects who did not receive the active antibiotics against MRSA, after confirming that the index blood culture was positive for MRSA. All patients were followed up until death or hospital discharge. If a patient had more than 1 episode of HA-MRSAB during the study period, only the first episode was included. Patients with polymicrobial bacteremia were excluded from the analysis specifically evaluating the influence of empirical antimicrobial therapy for HAMRSAB. Physicians treated patients according to routine medical practices without standardized protocols of intervention.

\section{Definitions}

Patients with healthcare-associated or nosocomial MRSAB were included in this study. MRSAB diagnosed within $48 \mathrm{~h}$ of hospital admission was considered healthcare-associated infection, if the patient met any healthcare-associated criteria in the preceding 3 months, including admission to a hospital, nursing home, or other healthcare facility for more than 2 days, specialized 
home care, treatment at a medical day unit, surgery, dialysis, or permanent indwelling catheters. MRSAB diagnosed after $48 \mathrm{~h}$ of hospital admission was considered nosocomial infection.

The primary focus of HA-MRSAB was determined by the organ affected and classified as follows: catheter-related bloodstream infection (CR-BSI) [22], lower respiratory tract, intra-abdominal, urinary tract, skin and soft tissue, bone and joint, or central nervous system infection; or unknown when no other site of infection was evident [23]. Sepsis, severe sepsis, and septic shock were diagnosed in accordance with standard criteria [24].

Definitive therapy was defined as the antibiotic therapy administered subsequent to the receipt of the final blood culture and antibiotic susceptibility results [17]. Empirical antibiotic therapy was defined as initial antibiotic treatment started before the pathogen was identified and antimicrobial susceptibility test results were obtained. Empirical antibiotic therapy was defined as appropriate if the pathogen was shown in in vitro studies to be susceptible to the antibiotic administered intravenously with optimal dosing and if the timing of administration was within $48 \mathrm{~h}$ of obtaining the index positive blood culture. If it did not meet any of these criteria, it was regarded as inappropriate antibiotic therapy. Prior antibiotic exposure was defined as the receipt of $>3$ doses of antibiotics within 3 months before the occurrence of HA-MRSAB. The duration of bacteremia after definitive antibiotic therapy was calculated as the number of days from initiation of definitive antibiotic treatment against HA-MRSAB to the day on which the first negative blood culture was obtained.

Mortality attributable to MRSA bacteremia was defined as death with positive blood cultures for MRSA and persistent fever, but no other definitive causes of death. Persistent MRSAB and persistent fever were defined as positive blood cultures for MRSA for $\geq 7$ days and as a fever $\geq 38.0{ }^{\circ} \mathrm{C}$ for $\geq 7$ days after the commencement of appropriate antibiotic treatment, respectively.

\section{Variables}

The clinical data of patients were extracted from medical records, including age, gender, medical history, comorbid medical conditions, Charlson's comorbidity index [25], primary site of infection, risk factors predisposing to infections within 3 months before the occurrence of HA-MRSAB, APACHE II scores [26], Pitt's bacteremia score [27] on the day of index positive blood culture sampling, presence of severe sepsis or septic shock, mortality attributable to MRSAB, and in-hospital mortality.

\section{Microbiological tests}

The identification of MRSA and antibiotic susceptibility tests were performed in each hospital using the MicroScan
Pos Combo Panel Type 6 automated system (Baxter Diagnostics, West Sacramento, CA, USA) or VITEK II automated system (bioMérieux, Hazelwood, MO, USA). All MRSA isolates from index blood cultures were collected at the coordinating center and immediately stored at $-70{ }^{\circ} \mathrm{C}$ until August 2012 when the MICs of vancomycin were further determined by the E-test (bioMérieux, Marcy l'Etoile, France) according to the manufacturer's instructions.

\section{Statistical analysis}

Categorical variables were denoted as count (proportion) and compared using Pearson's chi-squared test, Fisher's exact test, or McNemar's test. Continuous variables were expressed as the mean \pm standard deviation or median (inter-quartile range $[\mathrm{IQR}]$ ) and compared using twosample Student's $t$-tests, the Mann-Whitney U test, or Wilcoxon's signed-rank test. All tests were two-tailed, and a $P$-value $<0.05$ was considered statistically significant.

For the covariate adjustment between patients receiving inappropriate or appropriate empirical antibiotic therapy, variables that represented the probability of being treated inadequately were found. In univariate analysis of 345 patients with HA-MRSAB, baseline and clinical characteristics were compared between the two treatment groups. Variables at the $10 \%$ significance level were considered independent variables for the propensity scores, including category of infection, trauma, primary focus of HA-MRSAB (such as CR-BSI, pneumonia, or urinary tract infection), severe sepsis or septic shock, infective endocarditis, retention of foreign body, surgery, and recent exposure to immunosuppressive agents such as systemic corticosteroids on a regular basis or antineoplastic chemotherapy, third-generation cephalosporins, or fluoroquinolones during the preceding 30 days. Patients who received inappropriate empirical antibiotic therapy were matched 1:1 with patients who received appropriate empirical antibiotic therapy with regard to 5 digits of the propensity score without replacement $[28,29]$. To confirm whether we had a good match, the absolute standardized differences for each covariate in the model and distribution of propensity scores were evaluated [30]. To determine the predictors of in-hospital mortality in patients with HA-MRSAB, multivariate conditional logistic regression analyses within the matched data set were performed with all variables with a $P$-value $\leq 0.10$ in the univariate analysis as well as the main variable of inappropriate empirical antibiotic therapy.

Within the unmatched data set, inverse probability of treatment weighting (IPTW) based on the propensity score was also used to control for bias in treatment assignment [30, 31]. 
Data were analyzed using IBM SPSS Statistics version 20.0 (IBM Corporation, Armonk, NY, USA), R 2.15.2 (The R Foundation for Statistical Computing, Vienna, Austria), and SAS 9.2 (SAS Institute, Cary, NC, USA).

\section{Results}

During the study period, a total of 345 patients with HA-MRSAB were included in our analysis. Their demographic and clinical characteristics are presented in Table 1. The most common primary focus of HA-MRSAB was CR-BSI (51.3\%), followed by pneumonia (11.6\%), surgical wound infection (7.0\%), intra-abdominal infection $(21.0 \%)$, skin and soft tissue infection (4.9\%), and others $(4.2 \%)$. The all-cause in-hospital mortality and MRSAB-related mortality rates were $33.0(114 / 345)$ and
$16.5 \%$ (57/345), respectively. An in-hospital mortality rate of at least $25 \%$ was noted for pneumonia (55\%), central nervous system infections, MRSAB of unknown origin (44.4\%), intra-abdominal infections (38.1\%), CR-BSI $(33.3 \%)$, and cardiovascular infections (28.6\%), whereas mortality rates of less than $25 \%$ were observed for urinary tract infections, surgical wound infections, skin and soft tissue infections, bone and joint infections, and head and neck infections. Six patients died before the culture results were reported as follows: 4 patients presented with septic shock and 2 patients displayed high APACHE II scores of 30 and 39 , respectively.

Of the 345 patients with HA-MRSAB, 154 (44.6\%) patients received appropriate empirical antibiotic therapy. The antibiotics used for definitive therapy were vancomycin

Table 1 Demographic and clinical characteristics of 345 patients with healthcare-associated methicillin-resistant Staphylococcus aureus bacteremia according to the appropriateness of initial empirical antimicrobial therapy

\begin{tabular}{|c|c|c|c|c|}
\hline Variables & All $(n=345)$ & $\begin{array}{l}\text { Appropriate } \\
(n=154,44.6 \%)\end{array}$ & $\begin{array}{l}\text { Inappropriate } \\
(n=191,55.4 \%)\end{array}$ & $\begin{array}{l}\text { Odds ratio ( } 95 \% \\
\text { confidence interval) }\end{array}$ \\
\hline Male sex & $154(44.6)$ & $97(43.9)$ & $124(56.1)$ & $0.92(0.59-1.43)$ \\
\hline Age (years), median (IQR) & $67(52-75)$ & $66(51-74)$ & $67(53-75)$ & $0.99(0.98-1.01)$ \\
\hline \multicolumn{5}{|l|}{ Category of infection } \\
\hline Healthcare-associated ${ }^{a}$ & $51(14.8)$ & $29(18.8)$ & $22(11.5)$ & $1.78(0.98-3.25)$ \\
\hline Nosocomial & $294(85.2)$ & $125(81.2)$ & $169(88.5)$ & \\
\hline \multicolumn{5}{|l|}{ Comorbidity } \\
\hline Malignancy & $97(28.1)$ & $47(30.5)$ & $50(26.2)$ & $0.81(0.50-1.29)$ \\
\hline Metabolic & $122(35.4)$ & $52(33.8)$ & 70 (36.6) & $1.14(0.73-1.77)$ \\
\hline Trauma & $29(8.4)$ & $10(6.5)$ & $19(9.9)$ & $1.59(0.72-3.53)$ \\
\hline Charlson's comorbidity index ${ }^{\mathrm{b}}$, median (IQR) & $2(1-4)$ & $2(1-5)$ & $2(1-4)$ & $1.09(1.00-1.20)$ \\
\hline \multicolumn{5}{|l|}{ Primary focus of HA-MRSAB } \\
\hline CR-BSI & $177(51.3)$ & $93(60.4)$ & $84(44.0)$ & $0.52(0.34-0.79)$ \\
\hline Pneumonia & $40(11.6)$ & $13(8.4)$ & $27(14.1)$ & $1.79(0.89-3.59)$ \\
\hline \multicolumn{5}{|l|}{ Clinical severity } \\
\hline Development of severe sepsis or septic shock & $99(28.7)$ & $50(32.5)$ & $49(25.7)$ & $0.72(0.45-1.15)$ \\
\hline Pitt's bacteremia score at onset of bacteremia ${ }^{c}$, median (IQR) & $1(0-2)$ & $1(0-3)$ & $1(0-2)$ & $1.08(0.96-1.21)$ \\
\hline \multicolumn{5}{|l|}{ Predisposing factors } \\
\hline Surgical operation & $84(24.3)$ & $29(18.8)$ & $55(28.8)$ & $1.74(1.05-2.91)$ \\
\hline Foreign body retention & $15(4.3)$ & $12(7.8)$ & $3(1.6)$ & $0.189(0.05-0.69)$ \\
\hline Prior antibiotics use & $219(63.5)$ & $99(64.3)$ & $120(62.8)$ & $0.94(0.60-1.46)$ \\
\hline Vancomycin MIC, mg/L & $1(1-1.5)$ & $1(1-1.5)$ & $1(1-1.5)$ & $1.57(0.81-3.04)$ \\
\hline \multicolumn{5}{|l|}{ Outcomes } \\
\hline In-hospital mortality & $114(33.0)$ & $51(33.1)$ & $63(33.0)$ & $0.99(0.63-1.56)$ \\
\hline MRSAB-related mortality & $57(16.5)$ & $24(15.6)$ & $33(17.3)$ & $1.13(0.64-2.01)$ \\
\hline
\end{tabular}

IQR interquartile range, $A P A C H E$ acute physiology and chronic health evaluation, $C R-B S I$ catheter-related bloodstream infection, MIC minimum inhibitory concentration, MRSAB methicillin-resistant Staphylococcus aureus bacteremia ${ }^{a}$ MRSAB diagnosed within $48 \mathrm{~h}$ of hospital admission was considered healthcare-associated infection, if the patient presented with any healthcare-associated factor in the preceding 3 months

${ }^{b}$ Charlson's comorbidity score was calculated at the onset of MRSA bacteremia infection

'Pitt's bacteremia score and APACHE II scores were assessed at the onset of MRSA bacteremia 
( $n=201,58.3 \%)$, teicoplanin $(n=96,27.8 \%)$, arbekacin $(n=63,18.3 \%)$, linezolid $(n=34,9.9 \%)$, and tigecycline $(n=8,2.3 \%)$. Combination antibiotic therapy was administered to 15 patients as follows: glycopeptides plus rifampin $(n=11)$, linezolid plus arbekacin $(n=2)$, tigecycline plus arbekacin $(n=1)$, and teicoplanin plus arbekacin $(n=1)$.

For the non-matched case-control study $(n=345)$, the clinical outcomes of 154 patients who received appropriate empirical antibiotic therapy was compared with those of 191 patients who received inappropriate therapy. There were no significant differences in in-hospital mortality (odds ratio $[\mathrm{OR}]=0.99 ; 95 \%$ confidence interval $[\mathrm{CI}]=$ $0.63-1.56)$ or MRSAB-related mortality $(\mathrm{OR}=1.13$; $95 \%$ $\mathrm{CI}=0.64-2.01)$ between the two treatment groups (Table 1).

\section{Propensity score-matched analysis}

For the propensity score-matched (1:1) case-control study, only 127 patient pairs were matched according to the propensity score, because some variables, namely catheter-related bloodstream infection, retention of foreign body, surgical operation, and Charlson's comorbidity index, showed large differences between the two groups. Comparisons of demographic, clinical, and microbiological characteristics between the propensity-matched 127 pairs receiving inappropriate or appropriate empirical antibiotics are shown in Table 2. There were no significant differences in in-hospital mortality $(\mathrm{OR}=1.20 ; 95 \% \mathrm{CI}=$ $0.71-2.03)$ or MRSAB-related mortality $(\mathrm{OR}=1.34$; $95 \%$ $\mathrm{CI}=0.68-2.62)$ between the two treatment groups (Table 2). The antibiotics used for the appropriate empirical antibiotic therapy were vancomycin $(n=93,73.2 \%)$, teicoplanin $(n=22,17.3 \%)$, and arbekacin $(n=12,9.4 \%)$.

To assess the effect of empirical antibiotic therapy according to the clinical severity of HA-MRSAB, the clinical outcomes of patients with HA-MRSAB in association with empirical antibiotic therapy and the severity of HA-MRSAB were analyzed (Table 3 ). For the subgroup with severe sepsis $(n=75)$, septic shock $(n=45)$, or a higher Charlson's comorbidity index $(\geq 3)(n=108)$, there were no significant differences in either in-hospital mortality or MRSAB-related mortality between the patients receiving inappropriate or appropriate empirical antibiotic treatment (Table 3).

The covariate balance of independent variables for the propensity scores between the unmatched and matched data sets is presented in Table 4 . In the propensity scorematched analysis, matched standardized differences should be less than 0.20 and preferably 0.10 for the balanced distribution of the relevant variables between the two groups [32, 33]. Figure 1 demonstrates graphic overlays of distribution of the propensity scores before and after matching between the two treatment groups.
The factors associated with all-cause in-hospital mortality in patients with HA-MRSAB were determined in the propensity score-matched set. For the 254 patients included, demographic, clinical, and microbiological characteristics were compared between 84 non-survivors and 170 survivors (Table 2).

In multivariate conditional logistic regression analysis, severe sepsis or septic shock $(\mathrm{OR}=5.45$; $95 \% \mathrm{CI}=2.14$ 13.87), Charlson's comorbidity index (per 1-point increment; $\mathrm{OR}=1.52 ; 95 \% \mathrm{CI}=1.27-1.83$ ), and prior receipt of glycopeptides $(\mathrm{OR}=3.24 ; 95 \% \mathrm{CI}=1.08-9.67)$ were found to be independent risk factors for in-hospital mortality (Table 5).

As shown in Table 6, in multivariate logistic regression models of risk factors for in-hospital mortality using naïve, propensity score matching, or IPTW approaches, inappropriate empirical antibiotic therapy was not an independent predictor for mortality (Table 6).

\section{Microbiological factors and clinical outcomes}

A total of 296 (85.8 \%) MRSA isolates were available for analyzing vancomycin MICs using the E- test. The measured vancomycin MIC range was $0.5-2 \mathrm{mg} / \mathrm{L}$; vancomycin $\mathrm{MIC}_{50}$ and $\mathrm{MIC}_{90}$ were both $1.5 \mathrm{mg} / \mathrm{L}$. There was no difference in mortality outcomes between the two groups categorized as each reference cut-off of vancomycin $\mathrm{MICs}: \geq 1.0 \mathrm{mg} / \mathrm{L}(\mathrm{OR}=1.76 ; 95 \% \mathrm{CI}=$ 0.36-8.71), $\geq 1.5 \mathrm{mg} / \mathrm{L}(\mathrm{OR}=1.60 ; 95 \% \mathrm{CI}=0.91-2.83)$ and $\geq 2.0 \mathrm{mg} / \mathrm{L} \quad(\mathrm{OR}=2.46 ; 95 \quad \% \quad \mathrm{CI}=0.95-6.34)$ (Table 2).

\section{Discussion}

This propensity-matched study investigated the impact of inappropriate empirical antibiotic therapy on mortality after adjusting for potentially confounding factors that influence the receipt of inappropriate empirical antibiotic therapy in patients with HA-MRSAB in hospitals with a high prevalence of MRSA. This study found that an initial delay in the use of definitive antibiotics to which the MRSA isolates were susceptible did not necessarily prejudice the clinical outcomes of patients with HA-MRSAB and that the impetuous use of glycopeptide as empirical therapy targeting MRSA isolates should be moderated in terms of increasing antibiotic resistance.

Although initial empirical broad-spectrum antibiotic therapy, sometimes involving combination treatment, in bacteremic patients may appear to be an attractive treatment strategy before microbiological results are available, it can lead to increases in antibiotic resistance, costs, and adverse events. Studies investigating the association between inappropriate empirical antibiotic therapy and mortality among patients with bloodstream infections have reported conflicting findings. Thirteen studies have assessed the effects of empirical antibiotic 
Table 2 Comparison of clinical and microbiological characteristics and outcomes among patients with healthcare-associated methicillin-resistant Staphylococcus aureus bacteremia according to the appropriateness of empirical antibiotic therapy or treatment outcome in the propensity-matched analyses

\begin{tabular}{|c|c|c|c|c|c|c|c|}
\hline \multirow[t]{2}{*}{ Variables } & \multirow[t]{2}{*}{ Total } & \multicolumn{3}{|c|}{ Empirical antibiotic therapy } & \multicolumn{3}{|c|}{ Treatment outcome } \\
\hline & & $\begin{array}{l}\text { Inappropriate } \\
(n=127)\end{array}$ & $\begin{array}{l}\text { Appropriate } \\
(n=127)\end{array}$ & OR (95 \% Cl) & $\begin{array}{l}\text { Non-survival } \\
(n=81,31.9 \%)\end{array}$ & $\begin{array}{l}\text { Survival } \\
(n=173,68.1 \%)\end{array}$ & OR (95 \% Cl) \\
\hline Male sex & $165(65.0)$ & $84(66.1)$ & $81(63.8)$ & $1.03(0.62-1.72)$ & $59(70.2)$ & $106(62.4)$ & $0.66(0.38-1.15)$ \\
\hline Age (years), median (IQR) & $67(52-75)$ & $68(53-76)$ & $65(51-73)$ & $0.99(0.97-1.00)$ & $72(60-79)$ & $63(48-72)$ & $1.03(1.01-1.05)$ \\
\hline \multicolumn{8}{|l|}{ Category of infection } \\
\hline Healthcare-associated ${ }^{a}$ & $32(12.6)$ & $16(12.6)$ & $16(12.6)$ & $1.07(0.52-2.23)$ & $4(4.8)$ & $28(16.5)$ & $2.31(0.91-5.84)$ \\
\hline Nosocomial & $222(87.4)$ & $111(87.4)$ & $111(87.4)$ & & $80(95.2)$ & $142(83.5)$ & \\
\hline \multicolumn{8}{|l|}{ Comorbidity } \\
\hline Malignancy & $68(26.8)$ & $30(23.6)$ & $38(29.9)$ & $0.66(0.37-1.16)$ & $33(39.3)$ & 35 (20.6) & $2.63(1.46-4.73)$ \\
\hline Trauma & $24(9.4)$ & $14(11.0)$ & $10(7.9)$ & $1.33(0.56-3.17)$ & $7(8.3)$ & $17(10.0)$ & $0.93(0.37-2.35)$ \\
\hline Charlson's comorbidity index ${ }^{\mathrm{b}}$, median (IQR) & $2(1-4)$ & $2(1-4)$ & $2(1-4)$ & $1.08(0.97-1.19)$ & $3(2-6)$ & $2(0-2)$ & $1.37(1.21-1.54)$ \\
\hline \multicolumn{8}{|l|}{ Predisposing factors } \\
\hline Foreign body retention & $4(1.6)$ & $2(1.6)$ & $2(1.6)$ & $1.00(0.14-7.21)$ & $1(1.2)$ & $3(1.8)$ & $0.71(0.07-6.92)$ \\
\hline Surgical operation & $48(18.9)$ & $24(18.9)$ & $24(18.9)$ & $1.00(0.53-1.87)$ & $14(16.7)$ & $34(20.0)$ & $1.09(0.56-2.12)$ \\
\hline Prior antibiotic use & $151(59.4)$ & $73(57.5)$ & $78(61.4)$ & $1.00(0.60-1.66)$ & $58(69.0)$ & $93(54.7)$ & $2.39(1.33-4.30)$ \\
\hline Third-generation cephalosporins & $78(30.7)$ & $40(31.5)$ & $38(29.9)$ & $1.15(0.68-1.95)$ & $27(32.1)$ & $51(30.0)$ & $1.40(0.81-2.44)$ \\
\hline Fluoroquinolones & $39(15.4)$ & $17(113.4)$ & $22(17.3)$ & $0.89(0.45-1.75)$ & $16(19.8)$ & $23(13.5)$ & $1.96(0.99-3.91)$ \\
\hline Glycopeptides & $36(14.2)$ & $14(11.0)$ & $22(17.3)$ & $0.77(0.38-1.57)$ & $19(22.6)$ & $17(10.0)$ & $2.15(1.05-4.41)$ \\
\hline \multicolumn{8}{|l|}{ Primary focus of HA-MRSAB } \\
\hline CR-BSI & $144(56.7)$ & $72(56.7)$ & $72(56.7)$ & $1.03(0.63-1.70)$ & $52(61.9)$ & $92(54.1)$ & $1.03(0.61-1.75)$ \\
\hline Pneumonia & $22(8.7)$ & $12(9.4)$ & $10(7.9)$ & $0.41(0.62-3.21)$ & $11(13.1)$ & $11(6.5)$ & $3.35(1.46-7.67)$ \\
\hline \multicolumn{8}{|l|}{ Clinical severity } \\
\hline Development of severe sepsis or septic shock & $75(29.5)$ & $33(26.0)$ & $42(33.1)$ & $0.64(0.37-1.09)$ & $43(51.2)$ & $32(18.8)$ & $5.24(2.93-9.38)$ \\
\hline Pitt's bacteremia score', median (IQR) & $1(0-3)$ & $1(0-3)$ & $1(0-3)$ & $1.08(0.94-1.24)$ & $3(1-4)$ & $1(0-2)$ & $0 . .96(0.82-1.12)$ \\
\hline \multicolumn{8}{|l|}{ Vancomycin MIC, mg/L } \\
\hline $\mathrm{MIC} \geq 1.0 \mathrm{mg} / \mathrm{L}$ & $211(96.3)$ & $106(98.1)$ & $105(94.6)$ & $1.15(0.52-2.54)$ & $69(95.8)$ & $142(96.6)$ & $1.76(0.36-8.71)$ \\
\hline $\mathrm{MIC} \geq 1.5 \mathrm{mg} / \mathrm{L}$ & $94(42.9)$ & $39(36.1)$ & $55(49.5)$ & $0.60(0.31-1.16)$ & $34(47.2)$ & $60(40.8)$ & $1.60(0.91-2.83)$ \\
\hline $\mathrm{MIC} \geq 2 \mathrm{mg} / \mathrm{L}$ & $19(8.7)$ & $8(7.4)$ & $11(9.9)$ & $0.47(0.08-2.61)$ & $9(12.5)$ & $10(6.8)$ & $2.46(0.95-6.34)$ \\
\hline Appropriate empirical antibiotic therapy, $\mathrm{n}(\%)$ & $127(50.0)$ & & & & $42(50.0)$ & $85(50.0)$ & $1.20(0.71-2.03)$ \\
\hline \multicolumn{8}{|l|}{ Definitive therapy } \\
\hline Vancomycin & $145(57.1)$ & $68(53.5)$ & 77 (60.6) & $0.75(0.46-1.23)$ & $48(57.1)$ & $97(57.1)$ & $1.00(0.59-1.70)$ \\
\hline Teicoplanin & $75(29.5)$ & $38(29.9)$ & $37(29.1)$ & $1.04(0.61-1.78)$ & $26(31.0)$ & $49(28.8)$ & $1.11(0.63-1.96)$ \\
\hline Linezolid & $28(11.0)$ & $16(12.6)$ & $12(9.4)$ & $1.38(0.63-3.05)$ & $9(10.7)$ & $19(11.2)$ & $0.95(0.41-2.21)$ \\
\hline Arbekacin & $45(17.7)$ & $25(19.7)$ & $20(15.7)$ & $1.31(0.69-2.51)$ & $10(11.9)$ & 35 (20.6) & $0.52(0.24-1.11)$ \\
\hline Tigecycline & $4(1.6)$ & $2(1.6)$ & $2(1.6)$ & $1.00(0.14-6.99)$ & $2(2.4)$ & $2(1.2)$ & $2.05(0.28-14.80)$ \\
\hline Rifampin $^{d}$ & $9(3.5)$ & $7(5.5)$ & $2(1.6)$ & $3.65(0.74-17.9)$ & 0 & $9(5.3)$ & $0.95(0.91-0.98)$ \\
\hline \multicolumn{8}{|l|}{ Outcomes } \\
\hline In-hospital mortality & $84(33.1)$ & $42(33.1)$ & $42(33.1)$ & $1.20(0.71-2.03)$ & & & $1.20(0.71-2.03)$ \\
\hline MRSAB-related mortality & $40(15.7)$ & $21(16.5)$ & $19(15.0)$ & $1.34(0.68-2.62)$ & $40(47.6)$ & 0 & $2.03(1.62-2.53)$ \\
\hline
\end{tabular}

CR-BSI catheter-related bloodstream infection, IQR interquartile range, MIC minimum inhibitory concentration, MRSA methicillin-resistant Staphylococcus aureus, $M R S A B$ methicillin-resistant Staphylococcus aureus bacteremia

${ }^{a}$ MRSAB diagnosed within $48 \mathrm{~h}$ of hospital admission was considered healthcare-associated infection, if the patient presented with healthcare-associated factor in the preceding 3 months

${ }^{b}$ Charlson's comorbidity index was calculated at the first identification of MRSA bloodstream infection

'Pitt's bacteremia score was assessed at the first identification of MRSA bloodstream infection

${ }^{\mathrm{d}}$ Rifampin, which was prescribed in the survivals for the maintenance combination treatment during the finishing step for the small number of study cases 
Table 3 Comparison of in-hospital mortality rates according to the clinical severity of healthcare-associated methicillin-resistant Staphylococcus aureus bacteremia in the propensity-matched analyses

\begin{tabular}{|c|c|c|c|c|c|}
\hline & Empirical antibiotic therapy & In-hospital mortality, n (\%) & OR $(95 \% \mathrm{Cl})$ & MRSA-related mortality, n (\%) & OR $(95 \% \mathrm{Cl})$ \\
\hline \multicolumn{6}{|c|}{ Severe sepsis or septic shock $(n=75)$} \\
\hline \multirow[t]{2}{*}{ Yes } & Appropriate & $24(57.1)$ & $1.02(0.41-2.56)$ & $13(31.0)$ & $1.12(0.42-2.96)$ \\
\hline & Inappropriate & $19(57.9)$ & & $11(33.3)$ & \\
\hline \multirow[t]{2}{*}{ No } & Appropriate & $18(21.2)$ & $1.21(0.60-2.43)$ & $6(7.1)$ & $1.57(0.54-4.51)$ \\
\hline & Inappropriate & $23(24.5)$ & & $10(10.6)$ & \\
\hline \multicolumn{6}{|c|}{ Septic shock $(n=45)$} \\
\hline \multirow[t]{2}{*}{ Yes } & Appropriate & $20(66.7)$ & $1.00(0.27-3.72)$ & $12(40.0)$ & $1.00(0.28-3.54)$ \\
\hline & Inappropriate & $10(66.7)$ & & $6(40.0)$ & \\
\hline \multirow[t]{2}{*}{ No } & Appropriate & $22(22.7)$ & $1.36(0.73-2.55)$ & $7(7.2)$ & $1.99(0.78-5.10)$ \\
\hline & Inappropriate31 & $32(28.6)$ & & $15(13.4)$ & \\
\hline \multicolumn{6}{|c|}{ Charlson's comorbidity index $\geq 3(n=108)$} \\
\hline \multirow[t]{2}{*}{ Yes } & Appropriate & $31(56.4)$ & $0.62(0.29-1.32)$ & $13(23.6)$ & $0.83(0.33-2.05)$ \\
\hline & Inappropriate & $24(44.4)$ & & $11(20.8)$ & \\
\hline \multirow[t]{2}{*}{ No } & Appropriate & $11(15.3)$ & $1.82(0.79-4.18)$ & $6(8.3)$ & $1.75(0.60-5.09)$ \\
\hline & Inappropriate & $18(24.7)$ & & $10(13.7)$ & \\
\hline
\end{tabular}

MRSA methicillin-resistant Staphylococcus aureus

Table 4 Covariate balance of independent variables for the propensity scores between unmatched and matched data sets

\begin{tabular}{lll}
\hline & \multicolumn{2}{l}{$\begin{array}{l}\text { Absolute standardized } \\
\text { difference (\%) }\end{array}$} \\
\cline { 2 - 3 } & $\begin{array}{l}\text { Unmatched } \\
\text { data }\end{array}$ & $\begin{array}{l}\text { Matched } \\
\text { data }\end{array}$ \\
\hline Nosocomial infection & 20.49 & 0.00 \\
Trauma & 12.60 & 10.78 \\
Surgical operation & 23.56 & 0.00 \\
Immunosuppressive agents & 15.43 & 10.73 \\
Prior exposure to third-generation & 11.91 & 3.41 \\
cephalosporins & & \\
Prior exposure to fluoroquinolones & 12.36 & 10.94 \\
Prior exposure to glycopeptides & 14.06 & 18.14 \\
CR-BSI & 33.30 & 0.00 \\
Pneumonia & 18.07 & 5.60 \\
Urinary tract infection & 19.90 & 18.11 \\
Severe sepsis or septic shock & 15.05 & 15.58 \\
Retention of foreign body & 29.78 & 0.00 \\
Infective endocarditis & 15.25 & 18.11 \\
Charlson's comorbidity index & 21.35 & 16.12 \\
Pitt's bacteremia score & 13.35 & 11.08 \\
Age & 10.47 & 13.89 \\
Vancomycin MICs & 15.67 & 17.59 \\
\hline CRBSI cather & & \\
\hline
\end{tabular}

CR-BSI catheter-related bloodstream infection, MIC minimum inhibitory concentration therapy on mortality in study populations with MRSAB $[10-16,21,34-36]$. Seven of these study demonstrated that timely empirical therapy for MRSAB was associated with reduced mortality $[10,12-14,21,35,36]$. By contrast, six other studies reported an opposite result $[11,15,16,19,20,34]$. Interestingly, three studies conducted in Asia reported that an initial delay in the use of definitive antibiotics to which the MRSA isolates were susceptible did not have a detrimental effect on mortality $[11,19,20]$. Paul et al. [21] speculated that the studies did not confirm an advantage to empirical vancomycin treatment, as the prevalence of vancomycin MICs $>1.5 \mathrm{mg} / \mathrm{L}$ might be higher in Asia than in other locations [7, 9, 37-39].

On the contrary, a lack of agreement in the association between appropriate antibiotic therapy and mortality in bacteremic patients among the previous studies might be attributable to methodological heterogeneity. Appropriate antibiotic therapy and mortality were not consistently measured, and significant confounding variables were not always considered in the final analysis [17]. Therefore, in the present study on patients with HA-MRSAB, we obviously defined the appropriateness of antibiotic therapy in terms of its in vitro activity against the MRSA isolates, the timing and routes of administration, and the doses of the prescribed antibiotics; we also defined empirical and definitive antibiotic therapy clearly. We conducted multivariate analysis to adjust for confounding variables, including clinical severity and patients' comorbidity at the onset of HAMRSAB. Although the previous studies used a multivariate logistic regression analyses, residual confounding associated with the choice of appropriate or inappropriate 


\section{Before matching}

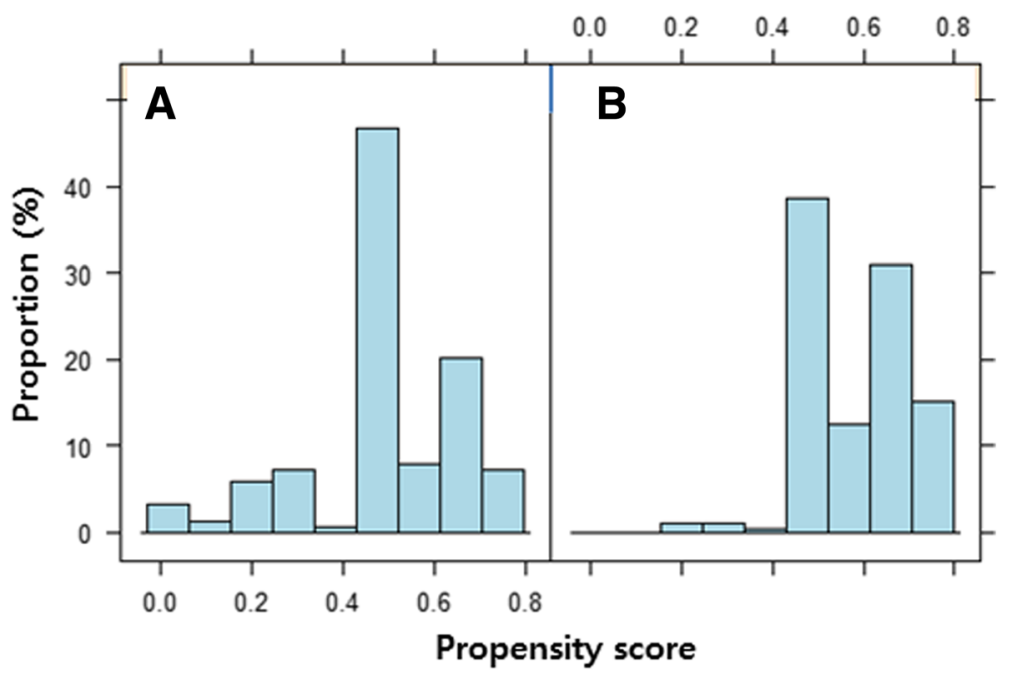

After matching

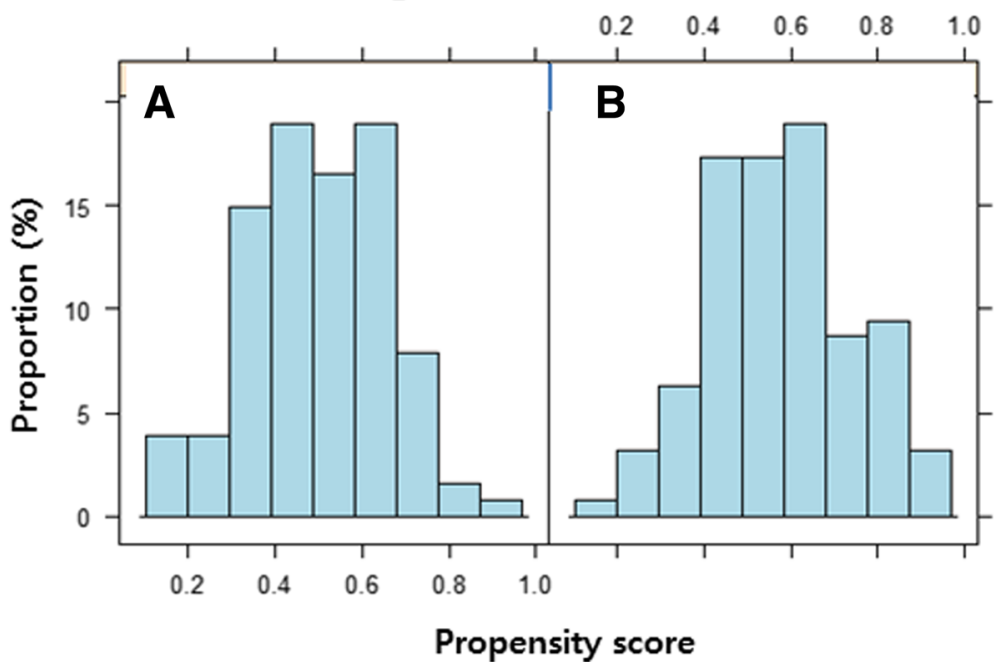

Fig. 1 The distribution of propensity scores in unmatched and matched data sets for the appropriate empirical antibiotic treatment (a) and inappropriate empirical antibiotic treatment groups (b)

empirical antibiotics was not fully excluded. Thus, our propensity-matched analysis calibrated all covariates that affect both the outcome and treatment assignment between patients who received appropriate and inappropriate empirical antibiotics.

In this study, there was no significant difference in mortality between the 127 pairs of propensity-matched patients who received inappropriate or appropriate empirical antibiotics. These results were comparable with the findings from a study by Kim et al, using a propensity score [20]. Among 345 patients with HAMRSAB analyzed in our study, empirical antibiotic therapy against MRSA isolates was given to 154 (44.6\%) patients. It was previously described that infections due to gram-positive organisms do not have a rapid course, unlike those caused by gram-negative pathogens $[40,41]$. In two meta-analyses of febrile neutropenic patients, there was no statistically significant difference in mortality between those who received a glycopeptide as part of the empirical regimen and those who did not receive glycopeptides [42, 43]. In addition, a large retrospective review from the National Cancer Institute suggested that the addition of vancomycin can be delayed until after four days of antibiotic monotherapy against Pseudomonas aeruginosa without any resulting increase in morbidity or mortality, even when a gram-positive infection was proven $[44,45]$. 
Table 5 Multivariate conditional logistic regression analysis of risk factors for in-hospital mortality in patients with healthcare-associated methicillin-resistant Staphylococcus aureus bacteremia in the propensity-matched analyses

\begin{tabular}{|c|c|c|c|c|c|c|}
\hline \multirow[b]{2}{*}{ Independent variable } & \multicolumn{2}{|c|}{$\begin{array}{l}\text { Unmatched data set } \\
(n=345)\end{array}$} & \multicolumn{2}{|c|}{$\begin{array}{l}\text { Unmatched data set } \\
\text { (IPTW, } n=345)\end{array}$} & \multicolumn{2}{|l|}{$\begin{array}{l}\text { Matched data set } \\
(n=254)\end{array}$} \\
\hline & OR $(95 \% \mathrm{Cl})$ & $P$-value & OR $(95 \% \mathrm{Cl})$ & $P$-value & OR $(95 \% \mathrm{Cl})$ & $P$-value \\
\hline Inappropriate empirical antibiotic therapy & $1.26(0.64-2.48)$ & 0.499 & $1.33(0.83-2.12)$ & 0.236 & $N A^{a}$ & 0.988 \\
\hline Nosocomial infection & $2.24(0.87-5.80)$ & 0.096 & $2.62(1.28-5.37)$ & 0.009 & & \\
\hline Trauma & $0.60(0.18-1.97)$ & 0.398 & $0.66(0.28-1.52)$ & 0.329 & $1.95(0.50-7.56)$ & 0.335 \\
\hline Surgical operation & $1.20(0.54-2.66)$ & 0.656 & $1.22(0.69-2.17)$ & 0.493 & & \\
\hline Prior exposure to third- generation cephalosporins & $1.34(0.68-2.63)$ & 0.399 & $1.23(0.76-2.00)$ & 0.407 & $0.93(0.41-2.10)$ & 0.853 \\
\hline Prior exposure to fluoroquinolones & $0.51(0.22-1.22)$ & 0.129 & $0.66(0.35-1.24)$ & 0.193 & $1.24(0.44-3.51)$ & 0.683 \\
\hline Prior exposure to glycopeptides & $2.85(1.20-6.77)$ & 0.018 & $2.86(1.55-5.28)$ & 0.001 & $3.24(1.08-9.67)$ & 0.035 \\
\hline CR-BSI & $0.92(0.44-1.92)$ & 0.825 & $0.79(0.47-1.32)$ & 0.360 & & \\
\hline Pneumonia & $0.37(0.14-1.00)$ & 0.050 & $0.28(0.14-0.59)$ & 0.001 & $2.15(0.51-9.04)$ & 0.296 \\
\hline Severe sepsis or septic shock & $3.84(1.85-7.96)$ & $<0.001$ & $3.64(2.17-6.10)$ & $<0.001$ & $5.45(2.14-13.87)$ & $<0.001$ \\
\hline Retention of foreign body & $1.63(0.40-6.71)$ & 0.500 & $1.96(0.62-6.18)$ & 0.250 & & \\
\hline Charlson's comorbidity index (per 1-point increment) & $0.66(0.57-0.77)$ & $<0.001$ & $0.68(0.61-0.75)$ & $<0.001$ & $1.52(1.27-1.83)$ & $<0.001$ \\
\hline Pitt's bacteremia score (per 1-point increment) & $0.77(0.64-0.93)$ & $<0.001$ & $0.76(0.66-0.87)$ & $<0.001$ & $1.23(0.99-1.54)$ & 0.067 \\
\hline Age (per 1-year increment) & $0.96(0.94-0.98)$ & 0.001 & $0.97(0.95-0.98)$ & $<0.001$ & $1.02(1.00-1.05)$ & 0.105 \\
\hline Vancomycin MICs & $0.48(0.20-1.16)$ & 0.103 & $0.42(0.23-0.79)$ & 0.007 & $1.55(0.52-4.62)$ & 0.433 \\
\hline
\end{tabular}

CR-BSI catheter-related bloodstream infection, IPTW inverse probability of treatment weighted, OR odds ratio, $95 \% \mathrm{Cl} 95 \%$ confidence interval, MIC minimum inhibitory concentration, NA not available

${ }^{a}$ These variables were not available because they displayed perfect marginal homogeneity with respect to each category concerning inappropriate initial empirical antibiotic therapy

In this study, the in-hospital mortality rate of HAMRSAB was $33.1 \%$, which is comparable to the range from $29 \%$ to $45 \%$ reported in other studies [10, 12, 16, 36]. Within our propensity score-matched data set, three prognostic factors were associated with in-hospital mortality in patients with HA-MRSAB: severe sepsis or septic shock, Charlson's comorbidity index at the onset of HA-MRSAB, and prior receipt of glycopeptides. These findings were similar to those of other studies [11-15, 20, 21]. In particular, prior exposure to glycopeptides was reported as an independent predictor of treatment failure for MRSAB in several studies [12, 13, $15,20,21]$. This fact strengthens the hypothesis that the empirical use of glycopeptides should be judicious even in patients suspected to have MRSAB. In addition, a higher vancomycin MIC has been recognized as one of the predictors associated with increased mortality and treatment failure among patients with MRSAB $[46,47]$. Prior exposure to glycopeptides is likely to be associated with mortality in patients with MRSAB, as recent exposure to vancomycin may lead to increased vancomycin MICs [48]. In this study, we failed to identify a significant impact of higher vancomycin MIC values on mortality in patients with HA-MRSAB, probably due to use of several antibiotics with activity against MRSAB. Vancomycin was used as a definite antibiotic in $58.3 \%$ of the 345 patients with HA-MRSAB analyzed in the study.

Table 6 Evaluation of the association between inappropriate empirical antibiotic therapy and in-hospital mortality in patients with healthcare-associated methicillin-resistant Staphylococcus aureus bacteremia

\begin{tabular}{|c|c|c|c|c|c|c|}
\hline & & \multirow[t]{2}{*}{ Number } & \multirow[t]{2}{*}{$P$-value } & \multirow[t]{2}{*}{ OR } & \multicolumn{2}{|c|}{$95 \% \mathrm{Cl}$} \\
\hline & & & & & $\mathrm{LL}$ & UL \\
\hline Model 1 & Unmatched sample, no adjustment & 345 & 0.979 & 1.01 & 0.64 & 1.58 \\
\hline Model 2 & Unmatched sample with no PS, adjusted for all covariates & 345 & 0.499 & 0.79 & 0.40 & 1.56 \\
\hline Model 3 & Unmatched sample with IPTW, adjusted for all covariates & 345 & 0.236 & 0.75 & 0.47 & 1.20 \\
\hline Model 4 & PS-matched sample, no adjustment & 254 & 0.984 & & $N A^{a}$ & \\
\hline Model 5 & PS-matched sample adjusted for all covariates & 254 & 0.988 & & $N A^{a}$ & . \\
\hline
\end{tabular}

PS propensity score, IPTW inverse probability of treatment weighted, OR odds ratio, $95 \%$ CI $95 \%$ confidence interval, LL lower limit, UL upper limit ${ }^{a}$ These variables were not available because they displayed perfect marginal homogeneity with respect to each category concerning inappropriate initial empirical antibiotic therapy 
There are some potential limitations in our study. First, the multivariate propensity-matched analysis still has a residual risk for confounding given the nonrandomized, controlled study design. Second, it should be acknowledged that culture sampling itself might have been delayed in some cases due to difficulties in suspecting the presence of MRSAB. Third, different kinds of antibiotics used for definitive therapy against MRSA can affect the clinical outcomes. However, there was no statistical difference in the distribution of definitive antibiotics in the comparative analyses of empirical antibiotic therapy or treatment outcomes. Fourth, vancomycin MIC for MRSA isolates was determined using the thawed, stored strains in this study, which can lead to an overall decline in vancomycin MICs measured for the same strains at the time of isolation. The effects of storage on the vancomycin MIC of the isolates may be considered confounders [49]. Lastly, this study showed a lack of significant difference in the hospital mortality between patients who received inappropriate or appropriate empirical antibiotics. This may imply an inconclusive result from the limited sample size, rather than a lack of any effect of appropriate empirical antibiotic therapy.

\section{Conclusions}

In conclusion, empirical inappropriate antibiotic therapy was not a significant predictor of mortality in patients with HA-MRSAB in hospitals with a high prevalence of MRSA. These findings suggest that it may be safe to await microbiological results and guide the use of definitive antibiotics in patients suspected of having HAMRSAB. Although earlier proper antibiotic prescribing is usually recommended for bacteremic patients by most experts, the empirical use of glycopeptides should be guided by the presence of predictors of MRSAB as well as the risk factors associated with MRSAB-related mortality.

\section{Abbreviations \\ CR-BSI, catheter-related bloodstream infection; HA-MRSAB, healthcare-associated methicillin-resistant Staphylococcus aureus bacteremia; IPTW, inverse probability of treatment weighting; IQR, inter-quartile range; MICs, minimum inhibitory concentrations; MRSA, methicillin-resistant Staphylococcus aureus; MRSAB, methicillin-resistant Staphylococcus aureus bacteremia}

\section{Acknowledgements}

None.

\section{Funding}

This study was financially supported in part by a grant (A102065) from the Korean Health 21 R\&D project, Ministry for Health, Welfare and Family Affairs, Republic of Korea, the City of Seoul Grant \#10920 and a National Research Foundation of Korea grant funded by the Korean government (No. K20902001448-10E0100-03010). The funders had no role in study design, data collection, data analysis, data interpretation, or writing the report.
Availability of data and materials

The data analyzed in this study can be accessed by sending a request to the corresponding author, after considering the progress of the ongoing post hoc analysis and with the approval of the Institutional Review Boards.

\section{Authors' contributions}

YKY performed the data verification, analyzed the data, and drafted the manuscript. MJK designed the study, and participated in manuscript revisions. KRP conceived the study. KSY conducted the statistical analysis. JYK performed the data verification and the integrity of the data. DWP, JWS, HYK YSK, CSL, MSL, SYR, HCJ, YJC, CIK, HJC, SSL, SWK, SIK, and ESK contributed clinical data. All authors read and approved the final manuscript.

\section{Competing interests}

The authors declare that they have no competing intersts.

\section{Consent for publication}

Not applicable.

\section{Ethics and consent to participate}

The study protocol was approved by the Institutional Review Board prior to starting the study at Korea University Anam Hospital (AN11209-002). As this observational study required no additional procedure apart from routine medical practice, the board waived the need for informed consent.

\section{Author details}

${ }^{1}$ Department of Internal Medicine, Korea University College of Medicine, Seoul, Republic of Korea. ${ }^{2}$ Department of Internal Medicine, Yonsei University Wonju College of Medicine, Won Ju, Republic of Korea. ${ }^{3}$ Department of Internal Medicine, Chungnam National University Hospital, Daejon, Republic of Korea. ${ }^{4}$ Department of Internal Medicine, Chonbuk National University Medical School, Jeonju, Republic of Korea. ${ }^{5}$ Department of Internal Medicine, Kyung Hee University School of Medicine, Seoul, Republic of Korea.

${ }^{6}$ Department of Internal Medicine, Keimyung University Dongsan Hospital, Daegu, Republic of Korea. ${ }^{7}$ Department of Internal Medicine, Chonnam National University Medical School, Gwangju, Republic of Korea. ${ }^{8}$ Department of Internal Medicine, National Cancer Center, Seoul, Republic of Korea. ${ }^{9}$ Department of Internal Medicine, Sungkyunkwan University School of Medicine, Seoul, Republic of Korea. ${ }^{10}$ Department of Internal Medicine, Ewha Women's University School of Medicine, Seoul, Republic of Korea.

${ }^{11}$ Department of Internal Medicine, Hallym University Sacred Heart Hospital, Anyang, Republic of Korea. ${ }^{12}$ Department of Internal Medicine, Kyungpook National University Hospital, Daegu, Republic of Korea. ${ }^{13}$ Department of Internal Medicine, Catholic University of Korea, College of Medicine, Seoul, Republic of Korea. ${ }^{14}$ Department of Internal Medicine, Seoul National University Bundang Hospital, Seoul, Republic of Korea. ${ }^{15}$ Department of Internal Medicine, Samyook Medical Center, Seoul, Republic of Korea.

${ }^{16}$ Department of Biostatistics, Korea University College of Medicine, Seoul, Republic of Korea.

Received: 12 November 2015 Accepted: 8 June 2016

Published online: 15 July 2016

\section{References}

1. Marra AR, Camargo LF, Pignatari AC, Sukiennik T, Behar PR, Medeiros EA, et al. Nosocomial bloodstream infections in Brazilian hospitals: analysis of 2,563 cases from a prospective nationwide surveillance study. J Clin Microbiol. 2011:49:1866-71.

2. Wisplinghoff $H$, Bischoff $T$, Tallent $S M$, Seifert $H$, Wenzel RP, Edmond MB. Nosocomial bloodstream infections in US hospitals: analysis of 24,179 cases from a prospective nationwide surveillance study. Clin Infect Dis. 2004:39:309-17.

3. Hall 2nd RG, Giuliano CA, Haase KK, Hazlewood KA, Frei CR, Forcade NA, et al. Empiric guideline-recommended weight-based vancomycin dosing and mortality in methicillin-resistant Staphylococcus aureus bacteremia: a retrospective cohort study. BMC Infect Dis. 2012;12:104

4. Moise-Broder PA, Sakoulas G, Eliopoulos GM, Schentag JJ, Forrest A, Moellering Jr RC. Accessory gene regulator group II polymorphism in methicillin-resistant Staphylococcus aureus is predictive of failure of vancomycin therapy. Clin Infect Dis. 2004;38:1700-5. 
5. Walraven CJ, North MS, Marr-Lyon L, Deming P, Sakoulas G, Mercier RC. Site of infection rather than vancomycin MIC predicts vancomycin treatment failure in methicillin-resistant Staphylococcus aureus bacteraemia. J Antimicrob Chemother. 2011;66:2386-92.

6. Rojas L, Bunsow E, Muñoz P, Cercenado E, Rodríguez-Créixems M, et al. Vancomycin MICs do not predict the outcome of methicillin-resistant Staphylococcus aureus bloodstream infections in correctly treated patients. J Antimicrob Chemother. 2012;67:1760-8.

7. Lin SH, Liao WH, Lai CC, Liao CH, Tan CK, Wang CY, et al. Risk factors for mortality in patients with persistent methicillin-resistant Staphylococcus aureus bacteraemia in a tertiary care hospital in Taiwan. J Antimicrob Chemother. 2010;65:1792-8.

8. Liao CH, Chen SY, Huang YT, Hsueh PR. Outcome of patients with methicillin-resistant Staphylococcus aureus bacteraemia at an Emergency Department of a medical centre in Taiwan. Int J Antimicrob Agents. 2008;32:326-32.

9. Wang JL, Wang JT, Sheng WH, Chen YC, Chang SC. Nosocomial methicillinresistant Staphylococcus aureus (MRSA) bacteremia in Taiwan: mortality analyses and the impact of vancomycin, $\mathrm{MIC}=2 \mathrm{mg} / \mathrm{L}$, by the broth microdilution method. BMC Infect Dis. 2010;10:159.

10. Gómez J, García-Vázquez E, Baños R, Canteras M, Ruiz J, Baños V, et al. Predictors of mortality in patients with methicillin-resistant Staphylococcus aureus (MRSA) bacteraemia: the role of empiric antibiotic therapy. Eur J Clin Microbiol Infect Dis. 2007:26:239-45.

11. Fang $C T$, Shau WY, Hsueh PR, Chen YC, Wang JT, Hung CC, et al. Early empirical glycopeptide therapy for patients with methicillin-resistant Staphylococcus aureus bacteraemia: impact on the outcome. J Antimicrob Chemother. 2006;57:511-9.

12. Marchaim D, Kaye KS, Fowler VG, Anderson DJ, Chawla V, Golan Y, et al. Case-control study to identify factors associated with mortality among patients with methicillin-resistant Staphylococcus aureus bacteraemia. Clin Microbiol Infect. 2010;16:747-52.

13. Rodríguez-Baño J, Millán AB, Domínguez MA, Borraz C, González MP, Almirante $B$, et al. Impact of inappropriate empirical therapy for sepsis due to health care-associated methicillin-resistant Staphylococcus aureus. J Infect. 2009;58:131-7.

14. Schramm GE, Johnson JA, Doherty JA, Micek ST, Kollef MH. Methicillinresistant Staphylococcus aureus sterile-site infection: The importance of appropriate initial antimicrobial treatment. Crit Care Med. 2006;34:2069-74.

15. Soriano A, Marco F, Martínez JA, Pisos E, Almela M, Dimova VP, et al. Influence of vancomycin minimum inhibitory concentration on the treatment of methicillin-resistant Staphylococcus aureus bacteremia. Clin Infect Dis. 2008:46:193-200.

16. Khatib R, Saeed S, Sharma M, Riederer K, Fakih MG, Johnson LB. Impact of initial antibiotic choice and delayed appropriate treatment on the outcome of Staphylococcus aureus bacteremia. Eur J Clin Microbiol Infect Dis. 2006;25:181-5.

17. McGregor JC, Rich SE, Harris AD, Perencevich EN, Osih R, Lodise Jr TP, et al. A systematic review of the methods used to assess the association between appropriate antibiotic therapy and mortality in bacteremic patients. Clin Infect Dis. 2007:45:329-37.

18. van Hal SJ, Jensen SO, Vaska VL, Espedido BA, Paterson DL, Gosbell IB. Predictors of mortality in Staphylococcus aureus Bacteremia. Clin Microbiol Rev. 2012;25:362-86

19. Lee $\mathrm{CH}$, Chien CC, Liu JW. Timing of initiating glycopeptide therapy for methicillin-Resistant Staphylococcus aureus bacteremia: the impact on clinical outcome. Scientific World Journal. 2013;2013:457435.

20. Kim SH, Park WB, Lee KD, Kang Cl, Bang JW, Kim HB, et al. Outcome of inappropriate initial antimicrobial treatment in patients with methicillin-resistant Staphylococcus aureus bacteraemia. J Antimicrob Chemother. 2004;54:489-97.

21. Paul M, Kariv G, Goldberg E, Raskin M, Shaked H, Hazzan R, et al. Importance of appropriate empirical antibiotic therapy for methicillin-resistant Staphylococcus aureus bacteraemia. J Antimicrob Chemother. 2010;65:2658-65.

22. Mermel LA, Allon M, Bouza E, Craven DE, Flynn P, O'Grady NP, et al. Clinical practice guidelines for the diagnosis and management of intravascular catheter-related infection: 2009 Update by the Infectious Diseases Society of America. Clin Infect Dis. 2009:49:1-45.

23. Horan TC, Andrus M, Dudeck MA. CDC/NHSN surveillance definition of health care-associated infection and criteria for specific types of infections in the acute care setting. Am J Infect Control. 2008;36:309-32.
24. Longo DL, Fauci AS, Kasper DL, Hauser SL, Jameson JL, Joseph L. Harrison's principles of Internal Medicine (ed 18). USA: Mc Graw Hill; 2008.

25. Charlson M, Szatrowski TP, Peterson J, Gold J. Validation of a combined comorbidity index. J Clin Epidemiol. 1994;47:1245-51.

26. Knaus WA, Draper EA, Wagner DP, Zimmerman JE. APACHE II: a severity of disease classification system. Crit Care Med. 1985:13:818-29.

27. Chow JW, Fine MJ, Shlaes DM, Quinn JP, Hooper DC, Johnson MP, et al. Enterobacter bacteremia: clinical features and emergence of antibiotic resistance during therapy. Ann Intern Med. 1991;115:585-90.

28. Rubin DB. Estimating causal effects from large data sets using propensity scores. Ann Intern Med. 1997;127:757-63.

29. Braitman LE, Rosenbaum PR. Rare outcomes, common treatments: analytic strategies using propensity scores. Ann Intern Med. 2002;137:693-5.

30. Austin PC. An introduction to propensity score methods for reducing the effects of confounding in observational studies. Multivariate Behav Res. 2011:46:399-424.

31. Sugihara M. Survival analysis using inverse probability of treatment weighted methods based on the generalized propensity score. Pharm Stat. 2010;9:21-34

32. Rosenbaum PR. Design of Observational Studies. New York: Springer; 2010

33. Lanza ST, Moore JE, Butera NM. Drawing causal inferences using propensity scores: a practical guide for community psychologists. Am J Community Psychol. 2013;52:380-92.

34. Schweizer ML, Furuno JP, Harris AD, Johnson JK, Shardell MD, McGregor JC, et al. Empiric antibiotic therapy for Staphylococcus aureus bacteremia may not reduce in-hospital mortality: a retrospective cohort study. PLoS One. 2010;5:e11432.

35. Lewis T, Chaudhry R, Nightingale P, Lambert P, Das I. Methicillin-resistant Staphylococcus aureus bacteremia: epidemiology, outcome, and laboratory characteristics in a tertiary referral center in the UK. Int J Infect Dis. 2011;15:e131-5.

36. Lodise TP, McKinnon PS, Swiderski L, Rybak MJ. Outcomes analysis of delayed antibiotic treatment for hospital-acquired Staphylococcus aureus bacteremia. Clin Infect Dis. 2003;36:1418-23.

37. Kim JS, Kim HS, Song W, Cho HC, Lee KM, Kim EC. Antimicrobial resistance profiles of Staphylococcus aureus isolated in 13 Korean hospitals. Korean J Lab Med. 2004;24:223-9.

38. Voss A, Doebbeling BN. The worldwide prevalence of methicillin-resistant Staphylococcus aureus. Int J Antimicrob Agents. 1995;5:101-6.

39. Takesue Y, Nakajima K, Takahashi Y, Ichiki K, Ishihara M, Wada Y, et al. Clinical characteristics of vancomycin minimum inhibitory concentration of $2 \mu \mathrm{g} / \mathrm{ml}$ methicillin-resistant Staphylococcus aureus strains isolated from patients with bacteremia. J Infect Chemother. 2011;17:52-7.

40. Feld R. Vancomycin as part of initial empirical antibiotic therapy for febrile neutropenia in patients with cancer: pros and cons. Clin Infect Dis. 1999:29:503-7.

41. Yu VL, Chiou CC, Feldman C, Ortqvist A, Rello J, Morris AJ, et al. An international prospective study of pneumococcal bacteremia: correlation with in vitro resistance, antibiotics administered, and clinical outcome. Clin Infect Dis. 2003;37:230-7.

42. Vardakas KZ, Samonis G, Chrysanthopoulou SA, Bliziotis IA, Falagas ME. Role of glycopeptides as part of initial empirical treatment of febrile neutropenic patients: a meta-analysis of randomised controlled trials. Lancet Infect Dis. 2005;5:431-9.

43. Paul M, Borok S, Fraser A, Vidal L, Leibovici L. Empirical antibiotics against Gram-positive infections for febrile neutropenia: systematic review and meta-analysis of randomized controlled trials. J Antimicrob Chemother. 2005;55:436-44

44. Pizzo PA, Hathorn JW, Hiemenz J, Browne M, Commers J, Cotton D, et al. A randomized trial comparing ceftazidime alone with combination antibiotic therapy in cancer patients with fever and neutropenia. N Engl J Med. 1986:315:552-8.

45. Rubin M, Hathorn JW, Marshall D, Gress J, Steinberg SM, Pizzo PA. Grampositive infections and the use of vancomycin in 550 episodes of fever and neutropenia. Ann Intern Med. 1988;108:30-5.

46. Jacob JT, DiazGranados CA. High vancomycin minimum inhibitory concentration and clinical outcomes in adults with methicillin-resistant Staphylococcus aureus infections: a meta-analysis. Int J Infect Dis. 2013;17:e93-e100

47. Mavros MN, Tansarli GS, Vardakas KZ, Rafailidis PI, Karageorgopoulos DE, Falagas ME. Impact of vancomycin minimum inhibitory concentration on 
clinical outcomes of patients with vancomycin-susceptible Staphylococcus aureus infections: a meta-analysis and meta-regression. Int J Antimicrob Agents. 2012;40:496-509.

48. Lubin AS, Snydman DR, Ruthazer R, Bide P, Golan Y. Predicting high vancomycin minimum inhibitory concentration in methicillin-resistant Staphylococcus aureus bloodstream infections. Clin Infect Dis. 2011;52:997-1002.

49. Ludwig F, Edwards B, Lawes T, Gould IM. Effects of storage on vancomycin and daptomycin MIC in susceptible blood isolates of methicillin-resistant Staphylococcus aureus. J Clin Microbiol. 2012;50:3383-7.

Submit your next manuscript to BioMed Central and we will help you at every step:

- We accept pre-submission inquiries

- Our selector tool helps you to find the most relevant journal

- We provide round the clock customer support

- Convenient online submission

- Thorough peer review

- Inclusion in PubMed and all major indexing services

- Maximum visibility for your research

Submit your manuscript at www.biomedcentral.com/submit
Biomed Central 